\title{
Herbig AeBe stars: Multiplicity and consequences
}

\author{
G. Duchêne ${ }^{1}$
}

\begin{abstract}
By virtue of their young age and intermediate mass, Herbig AeBe stars represent a cornerstone for our understanding of the mass-dependency of both the stellar and planetary formation processes. In this contribution, I review the current state-of-the-art multiplicity surveys of Herbig AeBe stars to assess both the overall frequency of companions and the distribution of key orbital parameters (separation, mass ratio and eccentricity). In a second part, I focus on the interplay between the multiplicity of Herbig AeBe stars and the presence and properties of their protoplanetary disks. Overall, it appears that both star and planet formation in the context of intermediate-mass stars proceeds following similar mechanisms as lower-mass stars.
\end{abstract}

Keywords Binaries: general; stars: early-type; stars: pre-main sequence

\section{Introduction}

Herbig AeBe (HAeBe) stars are young $(\lesssim 10 \mathrm{Myr})$, intermediate-mass $\left(1.5-8 M_{\odot}\right)$ stars whose defining characteristic is to host circumstellar protoplanetary disks Herbig 1960; Hillenbrand et al. 1992). As such, they provide an important perspective on the physics of stellar and planet formation. For one, they are higher mass counterparts to the well-studied Pre-Main Sequence (PMS) T Tauri stars (TTS). At the same time, they represent the initial stage of the formation of planetary systems around intermediate-mass stars, which have come in focus in recent years as it has become

G. Duchêne

Astronomy Department, University of California, Berkeley, CA 94720-3411 USA

${ }^{1}$ Univ. Grenoble Alpes, IPAG, F-38000 Grenoble, France CNRS, IPAG, F-38000 Grenoble, France clear that gas giant planets are even more common around intermediate-mass stars than they are around solar-type stars (e.g., Johnson et al. 2010).

It has long been known that stellar multiplicity is an ubiquitous phenomenon that is established during the star formation process itself (Mathieu 1994; Duchêne and Kraus 2013; Reipurth et al. 2014). Furthermore, there is a strong correlation between the multiplicity frequency and stellar mass on the Main Sequence (MS), so that single stars are a rare occurrence among field intermediate-mass stars (Abt 1983). The naive expectation is therefore that the multiplicity frequency of HAeBe stars is high. Indeed, the general population of intermediate-mass, mostly diskless, stars in the Sco-Cen OB association has a high multiplicity rate Kouwenhoven et al. 2007; Rizzuto et al. 2013). Among HAeBe stars, a high frequency of companion 11 has been found by a number of surveys in the past two decades (e.g., Leinert et al. 1997; Corporon and Lagrange 1999; Baines et al. 2006), albeit each within limited detectability ranges and possibly biased samples. Offering an updated view of this topic is one of the main goals of this contribution.

The presence of a close stellar companion can have serious implications on the formation of planetary systems. Examples of planets in a wide diversity of binary systems are known for solar-type stars, demonstrating that multiplicity and planet formation are not mutually exclusive (e.g., Raghavan et al. 2006; Bonavita and Desidera 2007; Kostov et al. 2014). However, not all multiple systems are equal in this regard. Visual binaries with separations tighter than 50-100 au

\footnotetext{
${ }^{1}$ Throughout this paper, the frequency of companions refers to the average number of companions per target, defined as $C F=$ $\frac{N_{2}+2 N_{3}+\ldots}{N_{1}+N_{2}+N_{3}+\ldots}$, where $N_{1}, N_{2}, N_{3}, \ldots$, represent to the number of single, binary, triple, ... systems, respectively. In the presence of many high-order systems, this quantity can exceed $100 \%$.
} 
are much less likely to host long-lived protoplanetary disks (e.g., Cieza et al. 2009) and the mature planets that orbit them have a markedly different mass distribution, suggesting that planet formation proceeds through a different mechanism than for wider systems and single stars (Duchêne 2010). Since HAeBe stars are selected based on the presence of a circumstellar disk, one may thus expect that they are less likely to possess close companions than a random sample of intermediate-mass stars.

The organization of this paper is as follows: Section2 presents an up-to-date overview of the frequency of multiple systems among HAeBe stars, Section 3 discusses the distribution of key orbital parameters, and Section 4 addresses the connection between multiple systems and disk properties among HAeBe stars. Finally, I discuss in Section 5 some of the implications from these findings and outline some directions for future studies.

\section{The multiplicity frequency of HAeBe stars}

\subsection{General remarks}

Fully assessing the multiplicity properties of a sample of stars is a considerable challenge, that has only been fully achieved for nearby solar-type field stars (Duquennoy and Mavor 1991; Raghavan et al. 2010). Given the extremely broad distribution of binary separations, thorough multiplicity surveys must combine several observing methods, each with its inherent limitations and selection biases. Furthermore, even with a comprehensive multi-technique approach, some ranges of separations can remain unexplored as a consequence of the relatively large distances to many targets. Consequently comparisons between various multiplicity surveys are often imperfect and limited to subsets of the parameter space, and/or require a simple parametrization of the underlying distribution of physical parameters (e.g., Kouwenhoven et al. 2007; Kraus et al. 2011).

These difficulties are exacerbated in the case of HAeBe stars. First of all, the scarcity of intermediatemass stars imposed by the stellar initial mass function (IMF) requires lumping HAeBe stars spanning a broad range of stellar masses into a single sample? even though this could smear out important but subtle trends. Furthermore, since most nearby star-forming regions only host a handful of intermediate-mass stars, most large samples of HAeBe stars are haphazard,

${ }^{2}$ Throughout this manuscript, I consider objects as late as earlyG stars as HAeBe stars, following the most common terminology in the literature (and in line with their stellar mass $M_{\star} \gtrsim 2 M_{\odot}$ ), even though these are sometimes referred to as TTS.

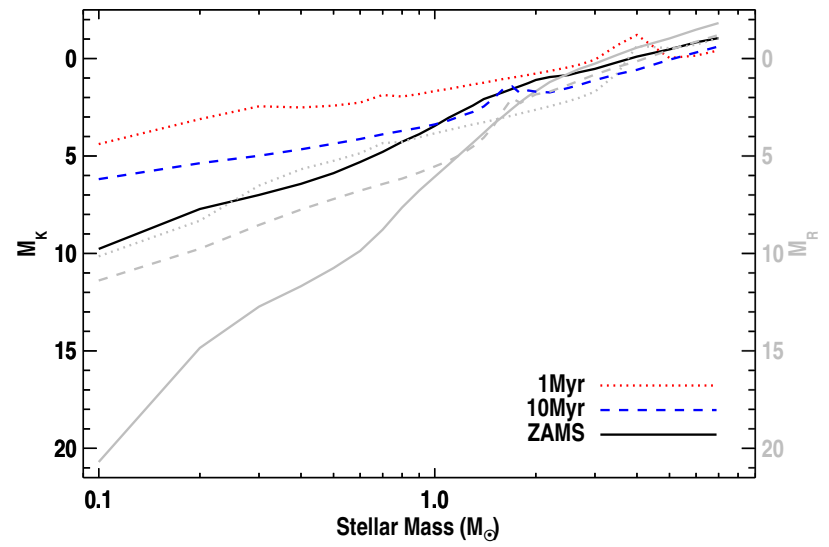

Fig. 1 Mass-luminosity relationships for PMS and ZAMS stars based on the evolutionary models of Siess et al. (2000). The $R$ and $K$ absolute magnitudes are shown with gray and colored lines, respectively.

bias-prone, mixed bags of stars with different evolutionary stages and spanning broad ranges of distances (The et al. 1994; Vieira et al. 2003; Hernández et al. 2005). Indeed, the majority of known HAeBe stars are located at least $300 \mathrm{pc}$ away from the Sun.

One issue that is often a severe challenge when searching for companions to intermediate-mass stars is the fact that the most unequal systems (with mass ratios $q=M_{2} / M_{1} \lesssim 0.1$ ) are characterized by large contrast ratios. As shown in Fig. [1 this is true if one considers the mass-luminosity relationship on the zeroage Main Sequence (ZAMS), which is relevant for field intermediate-mass stars whose age is typically a few $100 \mathrm{Myr}$. However, the situation is not nearly as severe for HAeBe, whose young ages ensure that their low-mass companions are still in the PMS phase. As a result, achieving a contrast of 5-6 mag in the nearinfrared is sufficient to detect the photosphere of any stellar companion to an HAeBe star. While this is a favorable circumstance, it also means that 1 ) determining the mass of a companion hinges on the ability to assess the age of the system, and 2) companions are likely to host their own circumstellar disk which can contribute significantly to its near-infrared brightness. At visible wavelengths, where thermal emission from the disk is negligible, the contrast between HAeBe stars and their low-mass companions remains prohibitively high, even at young ages. In summary, while detecting companions to HAeBe stars is easier than for their older field counterparts, accurately characterizing them remains challenging. 


\subsubsection{Visual binaries}

Over the last two decades, surveys for visual binaries among HAeBe stars have achieved continuously increasing resolution and contrast, as imaging techniques improved from direct imaging (Pirzkal et al. 1997; Doering and Meixner 2009) and speckle interferometry (Leinert et al. 1997) to adaptive optics (Bouvier and Cor 2001; Thomas et al. 2007). While sub-arcsecond companions are very likely to be bound, wider candidate companions require multi-epoch proper motion confirmation (e.g, Hornbeck et al. 2012). The most extensive and deepest survey to date has been conducted with adaptive optics by Thomas et al. (in prep.). Expanding the survey of Bouvier and Corporon (2001), their analysis includes 142 targets, a sample 3-5 times larger than those of Pirzkal et al. (1997) and (Leinert et al. 1997). In addition, where previous imaging searches were sensitive to companions up to 4-5mag fainter than their primary, the new adaptive optic surveys can detect companions up to 9 mag fainter (albeit with a significant dependence on separation within the central $\left.1^{\prime \prime}\right)$.

Based on the Thomas et al. survey, the observed companion frequency for HAeBe stars is about $25 \%$ per decade of separation in the $\approx 50-5000$ au range of projected separations. Note that this quantification is an effective way to deal with the diversity in distances to sources, since the distribution of separation of visual binaries is generally broad enough to be well approximated by a log-uniform distribution, i.e., Öpik's law (Öpik 1924). Not surprisingly, previous surveys led to lower companion frequencies (18-20\% per decade of separation), although the modest increase suggests that the frequency of (faint) very low-mass companions is modest.

Assessing the completeness of surveys, which depends not only on the separation-dependent sensitivity of surveys but also on the underlying (unknown) distribution of mass ratios and its possible dependence on binary separation, is a challenging endeavor that is beyond the scope of the present analysis. Wider separations cannot be probed because the proper motion of most HAeBe stars is too small to safely discriminate between background stars and bona fide physical companions. Furthermore, HAeBe stars are frequently surrounded by physically associated, but unbound, lowmass PMS stars at separations of thousands of au which formed from the same parent cloud (Testi et al. 1999).
Another technique to identify close visual companions is based on the detection of a spectro-astrometric signal. In a binary system comprising two stars of different effective temperature, the flux ratio of the binary varies significantly as a function of wavelength. The resulting displacements of the system's photocenter can be detected using long-slit spectroscopy. An inherent limi-

potan of this technique is its inability to determine precisely the binary separation (only a lower limit can be robustly established), except in cases for which a complete "deblending" analysis can be performed. Broadly speaking, the spectro-astrometry method is sensitive to companions as close as $\approx 0$ !' 1 and out to $2-5^{\prime \prime}$ (similar to the range probed with adaptive optics imaging, for instance), depending on seeing conditions and binary flux ratio. The companion frequency listed below applies within this approximative range.

Because it relies on spectral differences between the two components, this method is well adapted to the search of low-mass companions to HAeBe stars. Indeed, the spectro-astrometric method, which has targeted about $60 \mathrm{HAeBe}$ stars to date, has revealed a companion frequency for HAeBe stars as high as $\approx 75 \%$ (Baines et al. 2006; Wheelwright et al. 2010). This higher companion frequency than that found in direct imaging surveys may be the result of different sensitivity limits to low-mass companions. However, some candidate spectro-astrometric companions may be spurious as jets/outflows launched by the HAeBe star can also produce similar signatures. The suggestion that AB Aur is a binary system (e.g., Baines et al. 2006) whereas no other observing technique has found an actual stellar companion to that source (Liu et al. 2005; Perrin et al. 2009; Hashimoto et al. 2011) serves as a cautionary tale.

\subsubsection{Spectroscopic binaries}

While a number of spectroscopic binaries are known among HAeBe stars, systematic surveys remain few and far in between. Indeed, the combination of fast rotation, small number of photospheric features and strong emission lines is not particularly amenable to this technique. The incompleteness level of spectroscopic surveys depends on the complex interplay between the binary orbital period and mass ratio, the rotational velocity of the primary and the specific time sampling of the survey. Corporon and Lagrange (1999) estimated that their survey likely missed over half of all existing spectroscopic binaries in their sample, more than making up for any possible binary-favoring bias. It is 
worth stressing, however, that this estimate is highly dependent on currently untestable assumptions.

With these caveats in mind, Corporon and Lagrange (1999) found an observed companion frequency of about $30 \%$. Within a restricted range of orbital period where reasonable completeness can be ensured $(P \leq 100 \mathrm{~d})$, they derived a minimum companion frequency of $10 \%$. This is in line with the binary frequency estimated by Alecian et al. (2013), whose survey was not primarily designed for a multiplicity study and that had a very limited monitoring (for instance, only 11 out of their 70 targets have been observed more than twice). Thus, the companion frequency derived by Corporon and Lagrange (1999) is probably a more representative estimate.

Among spectroscopic binaries, the most remarkable systems are eclipsing binaries, which offer a unique chance of accurately determining both their mass and radius, thus providing critical tests for evolutionary models. The only well-studied such system is TY Cra, which actually is part of a compact triple system in which the eclipsing pair consists of a 1.5 and a $2.8 M_{\odot}$ stars, respectively Kardopolov et al. 1981; Corporon et al. 1994). Two more candidate systems have been proposed, T Ori and MWC 1080 (Grankin et al. 1992: Shevchenko and Vitrichenko 1994; Shevchenko et al. 1994; Corporon and Lagrange 1999), although no precise determination of the stellar parameters has been published to date.

\subsubsection{Intermediate separation binaries}

Spectroscopic binaries can only probe companions out to $\approx 1$ au given the long orbital period and small orbital velocity of wider systems. However, both the imaging and spectro-astrometric techniques are sensitive to companions whose separation is at least a few tens of au. This leaves a large "separation gap" in which the search for stellar companions can only be achieved with interferometric techniques: sparse aperture masking on large monolithic telescopes and long-baseline interferometry.

While no dedicated multiplicity survey has been conducted with either technique, many HAeBe stars have been ideal targets for both of them thanks to their intrinsic brightness. A literature search indicates that 56 HAeBe stars have been observed with at least one of the two techniques, resolving one triple system (GW Ori, Berger et al. 2011) and four binary systems (MWC 361, V892 Tau, V921 Sco, AK Sco; Millan-Gabet et al. 2001; Smith et al. 2005; Kraus et al. 2012, Anthonioz et al., in prep.). The resulting 11\% companion frequency should be considered as a lower limit given that the contrast afforded by interferometric techniques is generally modest and that only a handful of sources have been studied with both monolithic and long-baseline interferometric methods. Furthermore, faint stellar companions can be hard to distinguish if they lie at projected separations that are commensurate with the inner regions of the circumstellar disk, as the interferometric signatures of both features are interwoven.

\subsection{Towards a complete picture}

Although each of the survey methods discussed above has known limitations and (potentially insidious) selection biases, they nonetheless provide a nearly complete view of the multiplicity of HAeBe stars, at least out to separation of $\approx 5000 \mathrm{au}$. The overall companion frequency of HAeBe stars is at least $90 \%$, with the caveat that this estimate does not include any of the candidate spectro-astrometric companions that has not been confirmed by other methods. Considering the incompleteness of spectroscopic and interferometric surveys and the fact that field intermediate-mass stars host companions at separations as large as 45,000 au (De Rosa et al. 2014), it is most likely that there is at least one companion for each HAeBe star.

Such a high multiplicity frequency may explain why many HAeBe stars are strong X-ray emitters despite the fact their internal structure should not support the existence of a coherent stellar magnetic field (e.g., Zinnecker and Preibisch 1994). Indeed, it has long been proposed that the presence of a magnetically active, lower mass companion could account for this unexpected X-ray emission. However, the jury is still out as to whether this scenario applies to all cases or only a subset of the X-ray-detected HAeBe stars (Stelzer et al. 2009). In the latter case, the process leading to X-ray emission in single HAeBe stars remains to be identified.

\subsection{Comparison with other populations}

As mentioned above, it is highly valuable to compare the results of multiplicity studies of HAeBe stars discussed above to those of relevant stellar populations. Given the nature and scope of existing surveys, this is an exercise that is best performed by parts, however.

\subsubsection{Spectroscopic binaries}

There are too few spectroscopic binaries among HAeBe stars for a detailed analysis; only their overall frequency is reasonably well known at this point. The observed frequency of companions on short orbits (separation $\lesssim 1 \mathrm{au}$ ) is comparable to that observed for intermediate-mass stars in the field (Abt 1983). It also is higher than the corresponding frequency among TTS 


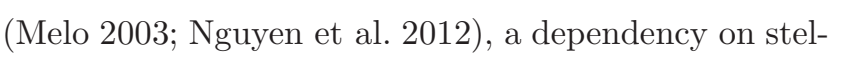
lar mass that is also observed among field stars. Thus current observations do not show significant deviations from expectations based on other stellar populations.

\subsubsection{Visual binaries}

The first surveys for visual binaries among populations of TTS revealed that wide binaries are more common in some star-forming regions than in others and, crucially, than is observed among field stars of similar masses (Duchêne 1999, and references therein). The population of HAeBe stars, which was poorly characterized for a long time, can now provide new insights on this topic.

Fig. [2 illustrates the frequency of visual companions for separations ranging from a few tens to $\sim 2000 \mathrm{au}$ among a variety of stellar populations as a function of their age and stellar mass. The companion frequency observed for HAeBe stars is similar to that of the (non-disk-bearing) intermediate-mass stars population in the Sco-Cen OB association, but higher than that of intermediate-mass field stars. Taking only into account the statistical (binomial) uncertainties, this excess is significant at the $3 \sigma$ level. However, it is currently impossible to evaluate the extent to which selection biases affect this estimate. The companion frequency of HAeBe stars is also marginally higher than that of lower mass stars in young loose associations like Taurus-Auriga, Chamaeleon and Upper Sco, which have the highest companion frequency of all populations of TTS.

\section{Other properties of HAeBe multiple systems}

Besides the overall frequency of companions, the distribution of orbital parameters (orbital period, mass ratio, eccentricity) and the relative frequency of binaries, triples and higher-order systems, are also rich diagnostics of the physical processes inherent to the formation and evolution of stellar systems. The incompleteness of current multiplicity surveys precludes statistical analyses of high-order systems among HAeBe stars, so I focus here on the distribution of orbital parameters.

\subsection{Orbital period distribution}

The distribution of separations for HAeBe binaries can be constructed by combining the surveys discussed above. The resulting distribution, shown in Fig.3, confirms that HAeBe stars have a companion frequency that is similar to that of field intermediate-mass stars

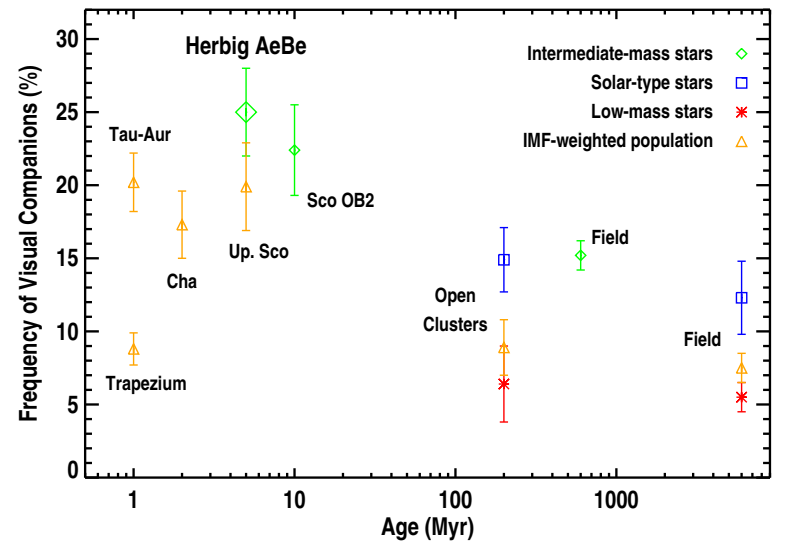

Fig. 2 Frequency of visual companion per decade of separation as a function of stellar age and mass. The frequencies are estimated for companions outside of $\sim 10 \mathrm{au}$ (50 au in the case of HAeBe stars and the Orion Trapezium), over a 1- to 2-decade-wide separation range depending on the sensitivity of existing surveys. The IMF-weighted populations are based on generic surveys of PMS stars for young populations $(\lesssim 10 \mathrm{Myr}$ ) and on an indicative $70 / 30$ split between low-mass and solar-type stars for older populations (0.1-0.5 and $0.6-1.5 M_{\odot}$, respectively). The companion frequencies shown here come from Bouvier et al. (1997, 2001); Delfosse et al. (2004); Kouwenhoven et al. (2005); Kraus et al. (2008); Kraus et al. (2011); Lafrenière et al. (2008); Reipurth et al. (2007); Reid and Gizis (1997); De Rosa et al. (2014); Raghavan et al. (2010), Thomas et al. (in prep.) and the latest results from the RECONS survey (http://www.recons.org). The ages used for open cluster and field populations are only representative as targets typically span a relatively wide age range within each category.

for short-period (spectroscopic) binaries and a significant excess for wide, visual binaries. In addition, it reveals an apparent minimum in the range probed by interferometric methods, which may also be associated with a deficit (by a factor of up to $\approx 2$ ) relative to field stars. This comparison must be undertaken with care, as this separation range is the least well determined for field stars (see, e.g., De Rosa et al. 2014). Furthermore, the sensitivity of interferometric surveys to stellar companions is the hardest to evaluate and plausibly the smallest among all techniques. Nonetheless, the reality of this deficit for HAeBe stars is further reinforced by the high companion frequency over this separation range for diskless B-type stars in the Sco-Cen association (Rizzuto et al. 2013). In summary, unless more than half of all stellar companions to HAeBe stars in that range remain undetected with interferometric techniques, both the minimum in the HAeBe separation distribution and the deficit of intermediate-separation systems relative to field stars are likely to be real. 


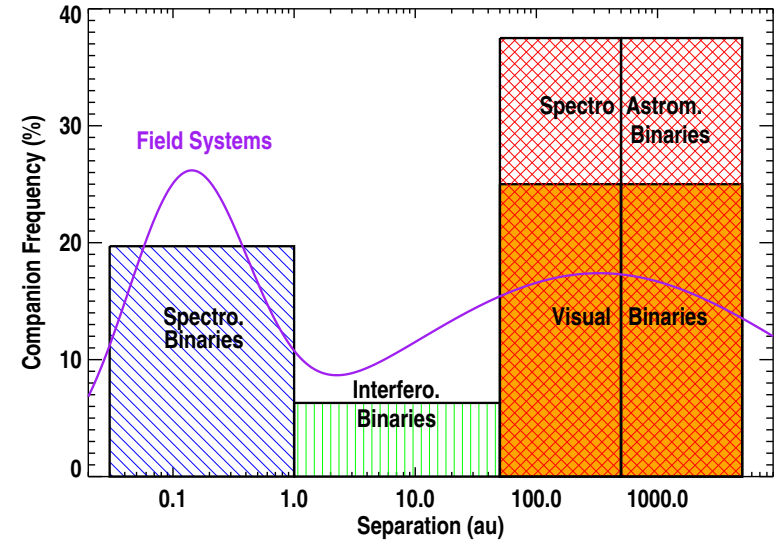

Fig. 3 Distribution of separation for HAeBe multiple systems. The vertical axis represents the companion frequency per decade of separation (as in Fig.2) over the approximate separation ranges probed by each technique, without any attempt to correct for incompleteness or overlap between them. Each technique is represented with a separate color and fill style. The purple curve is the schematic distribution for field A-type stars determined by Duchêne and Kraus (2013).

\subsection{Mass ratio distribution}

As discussed in Section 2.1, estimating masses for companions to HAeBe stars is not a straightforward matter. As a consequence, the mass ratio of each system is subject to significant uncertainties and comparing distributions derived from different methods may be fraught with systematic biases. It is nonetheless interesting to note that both the photometry-based distribution for visual binaries (Bouvier and Corporon 2001) and the spectroscopy-informed distribution for spectroastrometric binaries (Wheelwright et al. 2010) are reasonably consistent with one another. In short, the observed distributions are strongly inconsistent with an IMF-pairing of components in binary systems, but instead consistent with a roughly flat mass ratio distribution, at least down to $q \approx 0.2-0.4$, below which incompleteness is important. This is in line with observations of many populations of field stars (Duchêne and Kraus 2013), as well as of the populations of intermediatemass spectroscopic and visual binaries in the Sco-Cen OB association (Kouwenhoven et al. 2005, 2007) and those of TTS multiple systems (e.g., Kraus et al. 2011). Thus HAeBe binaries do not stand out among other populations of multiple systems as far as their mass ratio distribution is concerned.

\subsection{Eccentricity distribution}

The number of spectroscopic binaries among HAeBe stars is limited, and only a subset of these have had their orbit estimated. While this precludes any thorough statistical analysis of the overall distribution of eccentricities for this population, it is still informative to place all HAeBe binaries with published orbits in a period-eccentricity diagram, which has been extensively studied in the past (e.g., Abt 2005). To this end, I have compiled a list of 12 published spectroscopic orbits for $\mathrm{HAeBe}$ binaries that is complete to the best of my knowledge. Fig. (4 presents the resulting periodeccentricity diagram, along with those of disk-bearing TTS and non-disk-bearing B-type stars in the Sco-Cen OB association.

Broadly speaking, HAeBe binaries span similar distributions as field A- and B-type stars. Specifically, for orbital periods longer than $10 \mathrm{~d}$, eccentricities spanning most of the (0..1) range are found, similar to diskbearing TTS. Only three HAeBe binaries have shorter periods and all have near-circular orbits; the slightly non-zero eccentricity of the tight TY CrA AB pair is most likely a consequence of three-body interactions in this compact triple system (Beust et al. 1997). Give the scarcity of short period binaries, we can only conclude that the circularization period for HAeBe systems is in the $3-10 \mathrm{~d}$ range, similar to that of field A-type stars (e.g., Abt 2005). In turn, this suggests that circularization in intermediate-mass systems occurs on a timescale shorter than the typical age of HAeBe stars, hence $\lesssim 10$ Myr. This may be faster than previously believed (Abt et al. 2002), although uncertainties associated with tidal dissipation mechanisms remain large (e.g., Beust et al. 1997). Interestingly, 1-10 d B-type binaries in the Sco-Cen OB association have significantly higher eccentricities than HAeBe systems (see Fig.4) hinting at a significant mass-dependency for the tidal circularization, or at a causal link between disk survival and eccentricity of the central binary (i.e., a long-lived disk is able to circularize the binary orbit).

\section{HAeBe stars, multiplicity and disks}

With so many known HAeBe binaries, it is now possible to investigate the influence of multiplicity on their associated disks on empirical grounds. Here I discuss several key properties of HAeBe disks in the context of binary systems and, whenever possible, compare any trend with the situation of disks in lower-mass TTS binary systems. 


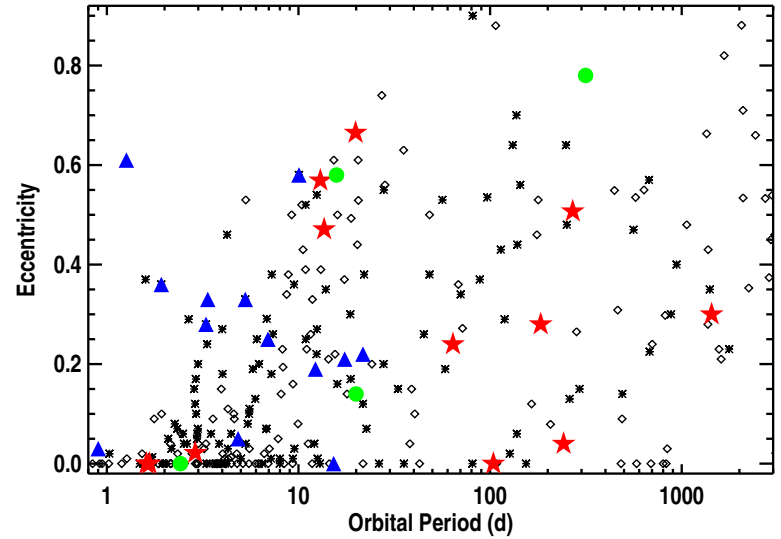

Fig. 4 Period-eccentricity diagram for spectroscopic binaries among HAeBe stars (filled red stars; Mathieu et al. 1991; Corporon et al. 1996; Corporon and Lagrange 1999; Alencar et al. 2003; Pogodin et al. 2006; Böhm et al. 2004, 2009; Alecian et al. 2009; Benisty et al. 2013; Beskrovnava et al. 2013), disk-bearing TTS (filled green circles; from the catalog of Ismailov et al. 2014) and nondisk-bearing B3- through B9-type stars in the Sco-Cen OB association (filled blue triangles; Levato et al. 1987; Brown and Verschueren 1997). The black diamonds and asterisks represent populations of field A- and B-type stars, respectively (Abt 2005).

\subsection{Disk-companion interactions}

\subsubsection{Influence of the binary separation}

First of all, the high frequency of companions observed among HAeBe stars is a clear confirmation that multiplicity and circumstellar disks are not mutually exclusive. However, the observed distribution of separations (Fig.3) suggests that companions at intermediate separations, roughly in the 1-50 au range, are less frequent among HAeBe stars than the overall population of intermediate-mass stars, a conclusion reminiscent of the results for TTS (Cieza et al. 2009; Kraus et al. 2012).

Given the breadth of the binary separation distribution and the typical size of protoplanetary disks $(\sim 100 \mathrm{au})$, HAeBe disks can be split into two distinct categories: circumstellar when the companion is a distant one, or circumbinary in the case of a tight pair. This diversity is also observed for TTS (e.g., Harris et al. 2012) as well as debris disks around solartype and intermediate-mass MS stars (Trilling et al. 2007; Rodriguez and Zuckerman 2012). As may have been expected, the presence of a companion to an HAeBe object therefore seems to have a very similar influence on its disk than for lower-mass primaries.

\subsubsection{HAeBe and transition disks}

Most circumbinary disks surround close, spectroscopic binaries. As pointed out above, slightly wider companions tend to completely disrupt the disk rather than simply perturbing it. There are exceptions to this rule, however, as revealed by the examples of the TTS systems GG Tau and UY Aur, for instance (e.g., Roddier et al. 1996; Close et al. 1998). Such systems may play an important role in the so-called "transition disks" phenomenon. Those were first identified among TTS as disks presenting massive mid- and farinfrared excesses but essentially no near-infrared excess Najita et al. 2007; Espaillat et al. 2014). This indicates that the innermost (hottest) regions of the disk have been cleared of dust, leaving only warm and cold dust further out. Several mechanisms can be responsible for this situation but one of them is the presence of a close (sub)stellar companion at a separation of a few $\mathrm{au}$, as demonstrated in the cases of CoKu Tau 4 and LkCa 15 Ireland and Kraus 2008; Kraus and Ireland 2012).

Since transition disks appear to be a common occurrence among HAeBe stars as well (e.g., Maaskant et al. 2013; Yasui et al. 2014), it is natural to wonder whether some of the large holes observed around young intermediatemass stars are carved by low-mass companions. Despite numerous searches for companions in HAeBe transition disks, the only confirmed such case to date is HD 142527, where a low-mass stellar companion has carved a large gap between the inner and outer regions of the disk (Biller et al. 2012; Close et al. 2014). It is therefore likely that the formation of a gap/hole in the inner regions of HAeBe disk is only rarely driven by the presence of a stellar companion, a similar conclusion as for TTS systems.

The focus has shifted in recent years toward planetarymass companions, which are now accessible thanks to improved contrast capabilities. A planetary mass companion has been proposed in the disk surrounding HD 100546 although it does not lie within a dust-empty region Quanz et al. 2013; Avenhaus et al.2014). More recently, a similar companion has been proposed to reside within the inner gap of the HD 169142 disk (Biller et al. 2014; Reggiani et al. 2014). Further observations are required to confirm the nature of this object and, more broadly, to test the hypothesis that newly-formed planets are responsible for the inner hole of transition disks.

\subsection{Disk-orbit relative orientation}

Depending on the formation scenario of the binary system, the disk midplane and the orbital plane can be 
tilted relative to one another. Therefore, the relative orientation of the disk and orbital planes is an important clue about the formation process of the system. Unfortunately, there are very few HAeBe binary systems in which both the orbit and disk orientation can be ascertained, the main limitation usually being the precise characterization of the orbital motion. Still, some HAeBe systems are amenable to individual studies thanks to their unique properties, as discussed below. Here I consider separately the cases of wide and close binaries, as they are probed via different methods.

\subsubsection{Circumstellar disks in wide binaries}

Most HAeBe visual binaries have orbits that are far too long to be derived, so it is usually not possible to determine the relative orientation of the orbit and the disk. There are however remarkable exceptions to this general rule. For instance, both the outflow and the binary orbit of the $\mathrm{LkH} \alpha 198$ system lie close to the plane of the sky, indicating that the circumstellar disk is nearly orthogonal to the binary orbit Smith et al. 2005). A strong argument can also be made in the PDS 144 system, where one of the two components has its disk viewed almost exactly edge-on while the other lies at a lower inclination, indicating that the two disks are misaligned by about $25^{\circ}$ (Perrin et al. 2006; Hornbeck et al. 2012). Necessarily, at least one of the two disks is misaligned with the (currently unknown) orbital plane.

Beyond individual systems, the only large-scale analysis of disk orientation to date has relied on spectropolarimetric determination of disk orientation and on a statistical treatment of the projection effects of the binary orbit and of its eccentricity. Using this method, Wheelwright et al. (2011) concluded that disks are not randomly oriented relative to their orbit (albeit only at the $2.2 \sigma$ confidence level), but instead that they are generally aligned with the binary orbital plane. However, it must be noted that this conclusion rests on the assumptions that most wide systems have low eccentricities. Instead, wide binaries among intermediate-mass field stars have an essentially uniform distribution of eccentricities, with an average eccentricity $\bar{e} \approx 0.5$ Abt 2005). If this also applies for HAeBe systems, the observed distribution of disk/orbit misalignment angles suggests that their disks only have a modest degree of alignment with the binary orbital plane (Fig. (5), i.e., the average angle between the orbital and disk plane in HAeBe binaries is small but non-zero (say, $\overline{\Delta i} \lesssim 30^{\circ}$ ).

While this is reminiscent of the conclusion that disks in TTS binary systems tend to be aligned with one another (Monin et al. 2007, and references therein), an

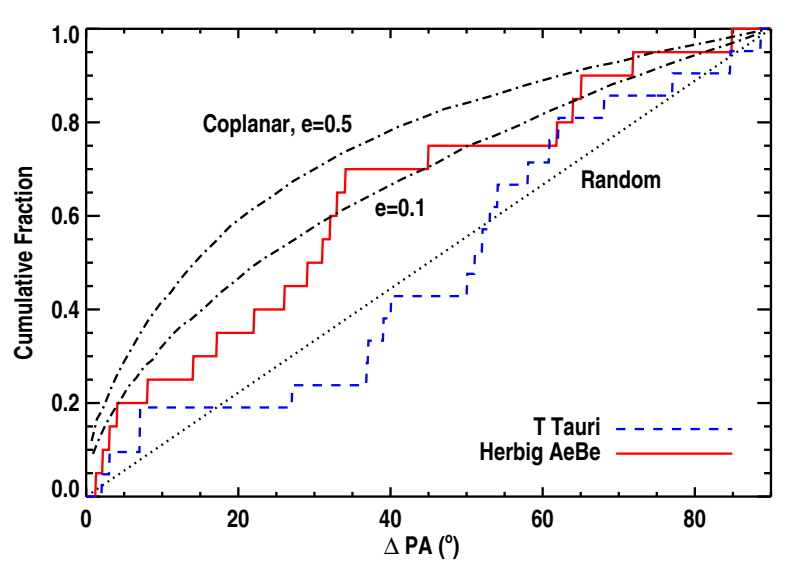

Fig. 5 Relative alignment of circumstellar disks in HAeBe and TTS wide binary systems (red and blue curves, respectively). The measured angle $(\Delta \mathrm{PA})$ is the difference in position angle between the disk semi-major axis and the projected separation of the binary system. The distribution for HAeBe systems is from Wheelwright et al. (2011) while data for TTS systems are from high-resolution millimeter imaging surveys (Andrews and Williams 2007; Andrews et al. 2010; Guilloteau et al. 2011; Harris et al. 2012; Akeson and Jensen 2014) after exclusion of systems in which the disk is too close to pole-on $\left(i \geq 30^{\circ}\right)$ or whose position angle is not known to better than $20^{\circ}$. The dotted and dot-dashed curves represent the expected distributions if the disk and orbital planes are randomly oriented and perfectly coplanar (for fixed eccentricities of $e=0.5$ and $e=0.1$; Wheelwright et al. 2011), respectively.

up-to-date distribution of disk-orbit misalignment angles based on recent high-resolution millimeter imaging surveys is most consistent with a completely random orientation for these disks (see Fig. (5) ). Furthermore, as for HAeBe systems, strongly misaligned disks have been observed in wide TTS binaries (e.g., Jensen and Akeson 2014). Overall, protoplanetary disks in low-mass binaries appear even more randomly oriented relative to their system's orbit than among HAeBe systems.

\subsubsection{Circumbinary disks around tight binaries}

While their orbits are conveniently short, spectroscopic orbital solutions are usually marred by an inclination ambiguity. This problem can be solved in cases where the system can be spatially resolved (typically through long-baseline interferometric observations). Detailed analyses have been possible in case of the GW Ori, HD 200775 and AK Sco systems (Vink et al. 2005; Berger et al. 2011; Benisty et al. 2013, Anthonioz et al., in prep.). In all three cases, it has been found that the disk, whose inner edge is located outside of the binary system, is (nearly) coplanar with 
the orbit. A possible counter-example is the V380 Ori system, where the spectroscopic binary has its orbital plane viewed pole-on (Alecian et al. 2009), while the giant outflow from the system propagates more or less in the plane of the sky Reipurth et al. (2013), hence the corresponding disk would be close to edge-on. However, it is possible that the outflow in this system arose from a violent dynamical reconfiguration, in whih case any directionality may not be indicative of the long-term disk orientation. Furthermore, it is not obvious that an outflow originating from a circumbinary disk should be exactly orthogonal to the latter, as the interaction with the inner system could easily break any symmetry. In any case, it appears that coplanarity for circumbinary disks is the rule in tight (spectroscopic and interferometric) HAeBe binaries.

Circumbinary disks among TTS systems display a similar degree of coplanarity, as illustrated by the cases of UZTau E and V4046 Sgr (Prato et al. 2002; Jensen et al. 2007; Rosenfeld et al. 2012). Interestingly, the widest TTS binary to host a circumbinary ring, the 35 au-separation GG Tau, has an orbit that is tilted by $15-25^{\circ}$ relative to the plane of the disk (Beust and Dutrey 2005; Köhler 2011), suggesting that near-perfect coplanarity only applies to systems whose semi-major axis does not exceed a few au.

\section{Implications and perspectives}

As I have shown in Section2 the initial assumption of a very high multiplicity of HAeBe stars is borne out by observations. With an average of at least one companion per HAeBe star, it is clear that multiplicity should not be considered a relatively rare phenomenon that can generally be ignored when studying an object in depth. Instead, low spatial resolution observations of objects whose multiplicity status has not yet been assessed should be interpreted with care. This is especially true of observations taken at wavelengths at which even a lower-mass companion can contribute significantly to the system's brightness, such as the farinfrared and millimeter ranges where disks around TTS companions can be quite bright. This may even be a factor to consider for future mid-infrared JWST observations of HAeBe stars. However, this high degree of multiplicity should not be interpreted as evidence that all HAeBe stars are members of binary systems either, as high-order multiple systems compensate for the small, but finite, fraction of single stars, akin to the situation of TTS (Kraus et al. 2011).

The main multiplicity properties of HAeBe stars, i.e., the companion frequency and distributions of orbital parameters, are mostly consistent with those observed for similarly young, but non-disk-bearing, intermediatemass stars in the Sco-Cen OB association, indicating that the HAeBe phenomenon is not very sensitive to the presence of a companion. While HAeBe stars host more companions overall than the lower-mass TTS, the two populations share roughly flat mass-ratio and eccentricity distributions (for $P \gtrsim 10 \mathrm{~d}$ ), suggesting that the star formation process proceeds through similar mechanisms over the entire $0.1-8 M_{\odot}$.

The apparent excess of visual companions for HAeBe stars over their MS counterparts is intriguing. Among populations of TTS, a similar excess has long been observed, and the debate as to whether this excess is indicative of intrinsic differences in the star formation process itself, or a consequence of intense dynamical evolution in young stellar clusters is still ongoing (e.g., King et al. 2012; Marks and Kroupa 2012). In any case, the excess has been interpreted as evidence that a majority of field stars form in dense clusters, in which wide companions are much less common (e.g., Patience et al. 2002; Koehler et al. 2006; Reipurth et al. 2007). It is unclear whether a similar line of thought can be applied to intermediate-mass stars, as HAeBe stars are distributed among both scattered and clustered populations, and thus do not represent a unique star-forming environment. The fact that intermediate-mass stars in the Sco-Cen association have a similar fraction of wide companions may instead suggest that this high companion frequency is universal. In turn, this would imply that a subset of these initial companions are in unstable configurations and are dispersed on a timescale of $\gtrsim 10 \mathrm{Myr}$. Probing the frequency of wide companions in populations of intermediate-mass stars in young open clusters $(\lesssim 100 \mathrm{Myr})$ would go a long way toward understanding this evolution. Unfortunately, the speckle interferometry survey of the $\alpha$ Per cluster by Patience et al. (2002) did not have sufficient sensitivity to low-mass stellar companions to be conclusive. New surveys using the high-contrast capabilities of planet-searching instruments (e.g., GPI, SCExAO, SPHERE) will provide a decisive input to this question.

As for lower mass PMS objects, the presence of a stellar companion does not appear to have dramatic effects on the circumstellar disks surrounding HAeBe stars, with the likely notable exception of companions at intermediate separations $(\approx 1-50 \mathrm{au})$. This can be readily understood as a companion in that separation range could dynamically disrupt any disk, or even prevent its formation altogether. It is worth pointing out, however, that when disks are present around Myr-old TTS in such binary systems, their lifetime is essentially the same as that of wider pairs and single stars 
(Kraus et al. 2012). Therefore, disks found in intermediate separation HAeBe binaries probably offer similar prospects to forming planetary systems as those around single stars, except that they are less common to begin with. In this context, it is worth restating that the transition disk phenomenon is in most cases not related to multiplicity. Instead, it is likely that the formation of a gap/hole in the inner regions of HAeBe disk is a consequence of similar disk evolution processes as for lower mass TTS. The combination of ALMA sub-mm observations with high contrast scattered light images is the most promising approach to understand the nature of these systems.

The diversity of architectures for disks in $\mathrm{HAeBe}$ multiple systems suggests that planet formation around intermediate-mass stars can lead to both circumstellar and circumbinary planets, as is observed for solartype stars. All planetary systems known among intermediate-mass stars have a circumstellar architecture but this is a consequence of the fact that they have only been searched via the radial velocity method (Johnson et al. 2010), whose precision is significantly limited in the case of close binary systems (Konacki et al. 2009). As the Kepler mission has shown, circumbinary planets are much easier to detect via the transit method, but the latter has not yet been employed much in the context of intermediatemass stars. Nonetheless, it is natural to expect that such systems exist and will be discovered in the future, for instance as part of the K2 phase of the Kepler mission.

Finally, the tentative near-perfect coplanarity of circumbinary disks around close, spectroscopic binaries (both among $\mathrm{HAeBe}$ and TTS) is in line with the configuration of the Kepler-discovered circumbinary planets around tight solar-type binaries Kostov et al. 2014). Astrometric monitoring of the orbit of spectroscopic HAeBe binaries with GAIA will likely increase manifold the number of systems in which this coplanarity can be tested. If confirmed, this coplanarity suggests a disk fragmentation origin for close binaries, although it is plausible that tidal torques can force at least the innermost region of the disk to settle in the same plane as the binary orbit if it is initially misaligned. Among wider systems, instead, circumstellar disks appear to be only moderately aligned with the orbital plane. This conclusion is reminiscent of the architecture of triple stellar systems (Hale 1994). Turbulent fragmentation of the parent cloud is the leading mechanism to generate such a configuration. In this scenario, the memory of the orientation of the cloud's angular momentum vector may only be partially erased from the various fragments. Furthermore, the fact that disks in TTS binaries are less aligned than those of HAeBe systems may indicate that the higher stellar masses of the latter are able to generate significant torques over timescales of a few Myr. Observations of the relative orientation of disks in the youngest (embedded) intermediate-mass binaries in the future would help in determining the exact initial configuration of the newly formed systems.

Acknowledgements Sandrine Thomas, Bernadette Rodgers, Fabien Anthonioz, Jérôme Bouvier, Adam Kraus, Rene Oudmaijer and an anonymous referee provided me with valuable feedback on various aspects of this work and shared some of their results in advance of publication, which I greatly appreciate. I am also grateful to the editors of the Topical Collection on HAeBe stars (Willem-Jan de Wit and Rene Oudmaijer) for inciting me to delve into this rich topic. 


\section{References}

Abt, H.A.: Annu. Rev. Astron. Astrophys. 21, 343 (1983) Abt, H.A.: Astrophys. J. 629, 507 (2005)

Abt, H.A., Levato, H., Grosso, M.: Astrophys. J. 573, 359 (2002)

Akeson, R.L., Jensen, E.L.N.: Astrophys. J. 784, 62 (2014)

Alecian, E., Wade, G.A., Catala, C., Bagnulo, S., Böhm, T., Bouret, J.-C., Donati, J.-F., Folsom, C.P., Grunhut, J., Landstreet, J.D.: Mon. Not. R. Astron. Soc. 400, 354 (2009)

Alecian, E., Wade, G.A., Catala, C., Grunhut, J.H., Landstreet, J.D., Bagnulo, S., Böhm, T., Folsom, C.P., Marsden, S., Waite, I.: Mon. Not. R. Astron. Soc. 429(2), 1001 (2013)

Alencar, S.H.P., Melo, C.H.F., Dullemond, C.P., Andersen, J., Batalha, C., Vaz, L.P.R., Mathieu, R.D.: Astron. Astrophys. 409, 1037 (2003)

Andrews, S.M., Williams, J.P.: Astrophys. J. 659, 705 (2007)

Andrews, S.M., Wilner, D.J., Hughes, A.M., Qi, C., Dullemond, C.P.: Astrophys. J. 723, 1241 (2010)

Avenhaus, H., Quanz, S.P., Meyer, M.R., Brittain, S.D., Carr, J.S., Najita, J.R.: Astrophys. J. 790, 56 (2014)

Baines, D., Oudmaijer, R.D., Porter, J.M., Pozzo, M.: Mon. Not. R. Astron. Soc. 367(2), 737 (2006)

Benisty, M., Perraut, K., Mourard, D., Stee, P., Lima, G.H.R.A., Le Bouquin, J.B., Borges Fernandes, M., Chesneau, O., Nardetto, N., Tallon-Bosc, I., McAlister, H., Ten Brummelaar, T., Ridgway, S., Sturmann, J., Sturmann, L., Turner, N., Farrington, C., Goldfinger, P.J.: Astron. Astrophys. 555, 113 (2013)

Berger, J.-P., Monnier, J.D., Millan-Gabet, R., Renard, S., Pedretti, E., Traub, W., Bechet, C., Benisty, M., Carleton, N., Haguenauer, P., Kern, P., Labeye, P., Longa, F., Lacasse, M., Malbet, F., Perraut, K., Ragland, S., Schloerb, P., Schuller, P.A., Thiebaut, E.: Astron. Astrophys. 529, 1 (2011)

Beskrovnaya, N.G., Pogodin, M.A., Valyavin, G.G., Ikhsanov N.R., Guseva, I.S., Pavlovskiy, S.E., Rusomarov, N., Ezhkova, O.V.: Astrophysics 56, 42 (2013)

Beust, H., Dutrey, A.: Astron. Astrophys. 439, 585 (2005)

Beust, H., Corporon, P., Siess, L., Forestini, M., Lagrange, A.-M.: Astron. Astrophys. 320, 478 (1997)

Biller, B.A., Males, J., Rodigas, T., Morzinski, K., Close, L.M., Juhász, A., Follette, K.B., Lacour, S., Benisty, M., Sicilia-Aguilar, A., Hinz, P.M., Weinberger, A., Henning, T., Pott, J.-U., Bonnefoy, M., Köhler, R.: ArXiv e-prints (2014). 1408.0794

Biller, B., Lacour, S., Juhász, A., Benisty, M., Chauvin, G., Olofsson, J., Pott, J.-U., Müller, A., Sicilia-Aguilar, A., Bonnefoy, M., Tuthill, P., Thébault, P., Henning, T., Crida, A.: Astrophys. J. 753(2), 38 (2012)

Böhm, T., Catala, C., Balona, L., Carter, B.: Astron. Astrophys. 427, 907 (2004)

Böhm, T., Zima, W., Catala, C., Alecian, E., Pollard, K., Wright, D.: Astron. Astrophys. 497, 183 (2009)

Bonavita, M., Desidera, S.: Astron. Astrophys. 468, 721 (2007)

Bouvier, J., Corporon, P.: In: Zinnecker, H., Mathieu, R. (eds.) The Formation of Binary Stars. IAU Symposium, vol. 200, p. 155 (2001)
Bouvier, J., Rigaut, F., Nadeau, D.: Astron. Astrophys. 323, 139 (1997)

Bouvier, J., Duchêne, G., Mermilliod, J.-C., Simon, T.: Astron. Astrophys. 375, 989 (2001)

Brown, A.G.A., Verschueren, W.: Astron. Astrophys. 319, 811 (1997)

Cieza, L.A., Padgett, D.L., Allen, L.E., McCabe, C.E., Brooke, T.Y., Carey, S.J., Chapman, N.L., Fukagawa, M., Huard, T.L., Noriga-Crespo, A., Peterson, D.E., Rebull, L.M.: Astrophys. J. Lett. 696, 84 (2009)

Close, L.M., Dutrey, A., Roddier, F., Guilloteau, S., Roddier, C., Northcott, M., Menard, F., Duvert, G., Graves, J.E., Potter, D.: Astrophys. J. 499, 883 (1998)

Close, L.M., Follette, K.B., Males, J.R., Puglisi, A., Xompero, M., Apai, D., Najita, J., Weinberger, A.J., Morzinski, K., Rodigas, T.J., Hinz, P., Bailey, V., Briguglio, R.: Astrophys. J. Lett. 781(2), 30 (2014)

Corporon, P., Lagrange, A.-M.: Astron. Astrophys. Suppl. Ser. 136, 429 (1999)

Corporon, P., Lagrange, A.M., Beust, H.: Astron. Astrophys. 310, 228 (1996)

Corporon, P., Lagrange, A.-M., Bouvier, J.: Astron. Astrophys. 282, 21 (1994)

De Rosa, R.J., Patience, J., Wilson, P.A., Schneider, A., Wiktorowicz, S.J., Vigan, A., Marois, C., Song, I., Macintosh, B., Graham, J.R., Doyon, R., Bessell, M.S., Thomas, S., Lai, O.: Mon. Not. R. Astron. Soc. 437(2), 1216 (2014)

Delfosse, X., Beuzit, J.-L., Marchal, L., Bonfils, X., Perrier, C., Ségransan, D., Udry, S., Mayor, M., Forveille, T.: In: Hilditch, R.W., Hensberge, H., Pavlovski, K. (eds.) Spectroscopically and Spatially Resolving the Components of the Close Binary Stars. Astronomical Society of the Pacific Conference Series, vol. 318, p. 166 (2004)

Doering, R.L., Meixner, M.: Astron. J. 138(3), 780 (2009)

Duchêne, G.: Astron. Astrophys. 341, 547 (1999)

Duchêne, G.: Astrophys. J. Lett. 709(2), 114 (2010)

Duchêne, G., Kraus, A.: Annu. Rev. Astron. Astrophys. 51, 269 (2013)

Duquennoy, A., Mayor, M.: Astron. Astrophys. 248, 485 (1991)

Espaillat, C., Muzerolle, J., Najita, J., Andrews, S., Zhu, Z., Calvet, N., Kraus, S., Hashimoto, J., Kraus, A., D'Alessio, P.: ArXiv e-prints (2014). 1402.7103

Grankin, K.N., Shevchenko, V.S., Chernyshev, A.V., Ibragimov, M.A., Kondratiev, W.B., Melnikov, S.Y., Yakubov, S.D., Melikian, N.D., Abramian, G.V.: Information Bulletin on Variable Stars 3747, 1 (1992)

Guilloteau, S., Dutrey, A., Piétu, V., Boehler, Y.: Astron. Astrophys. 529, 105 (2011)

Hale, A.: Astron. J. 107, 306 (1994)

Harris, R.J., Andrews, S.M., Wilner, D.J., Kraus, A.L.: Astrophys. J. 751, 115 (2012)

Hashimoto, J., Tamura, M., Muto, T., Kudo, T., Fukagawa, M., Fukue, T., Goto, M., Grady, C.A., Henning, T., Hodapp, K., Honda, M., Inutsuka, S., Kokubo, E., Knapp, G., McElwain, M.W., Momose, M., Ohashi, N., Okamoto, Y.K., Takami, M., Turner, E.L., Wisniewski, J., Janson, M., Abe, L., Brandner, W., Carson, J., Egner, S., Feldt, M., Golota, T., Guyon, O., Hayano, Y., Hayashi, M., 
Hayashi, S., Ishii, M., Kandori, R., Kusakabe, N., Matsuo, T., Mayama, S., Miyama, S., Morino, J.-I., MoroMartín, A., Nishimura, T., Pyo, T.-S., Suto, H., Suzuki, R., Takato, N., Terada, H., Thalmann, C., Tomono, D., Watanabe, M., Yamada, T., Takami, H., Usuda, T.: Astrophys. J. Lett. 729(2), 17 (2011)

Herbig, G.H.: Astrophys. J. Suppl. Ser. 4, 337 (1960)

Hernández, J., Calvet, N., Hartmann, L., Briceño, C., Sicilia-Aguilar, A., Berlind, P.: Astron. J. 129, 856 (2005)

Hillenbrand, L.A., Strom, S.E., Vrba, F.J., Keene, J.: Astrophys. J. 397, 613 (1992)

Hornbeck, J.B., Grady, C.A., Perrin, M.D., Wisniewski, J.P., Tofflemire, B.M., Brown, A., Holtzman, J.A., Arraki, K., Hamaguchi, K., Woodgate, B., Petre, R., Daly, B., Grogin, N.A., Bonfield, D.G., Williger, G.M., Lauroesch, J.T.: Astrophys. J. 744(1), 54 (2012)

Ireland, M.J., Kraus, A.L.: Astrophys. J. Lett. 678, 59 (2008)

Ismailov, N.Z., Abdi, H.A., Mamedxanova, G.B.: Astronomicheskij Tsirkulyar 1610, 1 (2014)

Jensen, E.L.N., Akeson, R.: Nature 511, 567 (2014)

Jensen, E.L.N., Dhital, S., Stassun, K.G., Patience, J., Herbst, W., Walter, F.M., Simon, M., Basri, G.: Astron. J. 134, 241 (2007)

Johnson, J.A., Aller, K.M., Howard, A.W., Crepp, J.R.: Publ. Astron. Soc. Pac. 122, 905 (2010)

Kardopolov, V.I., Sakhanenok, V.V., Filip'ev, G.K.: Peremennye Zvezdy 21, 589 (1981)

King, R.R., Goodwin, S.P., Parker, R.J., Patience, J.: Mon. Not. R. Astron. Soc. 427, 2636 (2012)

Koehler, R., Petr-Gotzens, M.G., McCaughrean, M.J., Bouvier, J., Duchene, G., Quirrenbach, A., Zinnecker, H.: Astron. Astrophys. 458, 461 (2006)

Köhler, R.: Astron. Astrophys. 530, 126 (2011)

Konacki, M., Muterspaugh, M.W., Kulkarni, S.R., Hełminiak, K.G.: Astrophys. J. 704, 513 (2009)

Kostov, V.B., McCullough, P.R., Carter, J.A., Deleuil, M., Díaz, R.F., Fabrycky, D.C., Hébrard, G., Hinse, T.C., Mazeh, T., Orosz, J.A., Tsvetanov, Z.I., Welsh, W.F.: Astrophys. J. 784, 14 (2014)

Kouwenhoven, M.B.N., Brown, A.G.A., Zinnecker, H., Kaper, L., Portegies Zwart, S.F.: Astron. Astrophys. 430, 137 (2005)

Kouwenhoven, M.B.N., Brown, A.G.A., Portegies Zwart, S.F., Kaper, L.: Astron. Astrophys. 474, 77 (2007)

Kraus, A.L., Ireland, M.J.: Astrophys. J. 745, 5 (2012)

Kraus, A.L., Ireland, M.J., Martinache, F., Lloyd, J.P.: Astrophys. J. 679, 762 (2008)

Kraus, A.L., Ireland, M.J., Hillenbrand, L.A., Martinache, F.: Astrophys. J. 745, 19 (2012)

Kraus, A.L., Ireland, M.J., Martinache, F., Hillenbrand, L.A.: Astrophys. J. 731(1), 8 (2011)

Kraus, S., Calvet, N., Hartmann, L., Hofmann, K.-H., Kreplin, A., Monnier, J.D., Weigelt, G.: Astrophys. J. Lett. 746(1), 2 (2012)

Lafrenière, D., Jayawardhana, R., Brandeker, A., Ahmic, M., van Kerkwijk, M.H.: Astrophys. J. 683, 844 (2008)

Leinert, C., Richichi, A., Haas, M.: Astron. Astrophys. 318, 472 (1997)
Levato, H., Malaroda, S., Morrell, N., Solivella, G.: Astrophys. J. Suppl. Ser. 64, 487 (1987)

Liu, W.M., Hinz, P.M., Hoffmann, W.F., Brusa, G., Miller, D., Kenworthy, M.A.: Astrophys. J. 618, 133 (2005)

Maaskant, K.M., Honda, M., Waters, L.B.F.M., Tielens, A.G.G.M., Dominik, C., Min, M., Verhoeff, A., Meeus, G., van den Ancker, M.E.: Astron. Astrophys. 555, 64 (2013)

Marks, M., Kroupa, P.: Astron. Astrophys. 543, 8 (2012)

Mathieu, R.D., Adams, F.C., Latham, D.W.: Astron. J. 101, 2184 (1991)

Mathieu, R.D.: Annu. Rev. Astron. Astrophys. 32, 465 (1994)

Melo, C.H.F.: Astron. Astrophys. 410, 269 (2003)

Millan-Gabet, R., Schloerb, F.P., Traub, W.A.: Astrophys. J. 546, 358 (2001)

Monin, J.-L., Clarke, C.J., Prato, L., McCabe, C.: Protostars and Planets V, 395 (2007). astro-ph/0604031

Najita, J.R., Strom, S.E., Muzerolle, J.: Mon. Not. R. Astron. Soc. 378, 369 (2007)

Nguyen, D.C., Brandeker, A., van Kerkwijk, M.H., Jayawardhana, R.: Astrophys. J. 745, 119 (2012)

Öpik, E.: Publications of the Tartu Astrofizica Observatory 25, 1 (1924)

Patience, J., Ghez, A.M., Reid, I.N., Matthews, K.: Astron. J. 123, 1570 (2002)

Perrin, M.D., Duchêne, G., Kalas, P., Graham, J.R.: Astrophys. J. 645, 1272 (2006)

Perrin, M.D., Schneider, G., Duchene, G., Pinte, C., Grady, C.A., Wisniewski, J.P., Hines, D.C.: Astrophys. J. Lett. 707(2), 132 (2009)

Pirzkal, N., Spillar, E.J., Dyck, H.M.: Astrophys. J. 481, 392 (1997)

Pogodin, M.A., Malanushenko, V.P., Kozlova, O.V., Tarasova T.N., Franco, G.A.P.: Astron. Astrophys. 452, 551 (2006)

Prato, L., Simon, M., Mazeh, T., Zucker, S., McLean, I.S.: Astrophys. J. Lett. 579, 99 (2002)

Quanz, S.P., Amara, A., Meyer, M.R., Kenworthy, M.A., Kasper, M., Girard, J.H.: Astrophys. J. Lett. 766, 1 (2013)

Raghavan, D., Henry, T.J., Mason, B.D., Subasavage, J.P., Jao, W.-C., Beaulieu, T.D., Hambly, N.C.: Astrophys. J. 646, 523 (2006)

Raghavan, D., McAlister, H.A., Henry, T.J., Latham, D.W., Marcy, G.W., Mason, B.D., Gies, D.R., White, R.J., ten Brummelaar, T.A.: Astrophys. J. Suppl. Ser. 190, 1 (2010)

Reggiani, M., Quanz, S.P., Meyer, M.R., Pueyo, L., Absil, O., Amara, A., Anglada, G., Avenhaus, H., Girard, J.H., Carrasco Gonzalez, C., James, G., Mawet, D., Meru, F., Milli, J., Osorio, M., Wolff, S., Torrelles, J.-M.: ArXiv e-prints (2014). 1408.0813

Reid, I.N., Gizis, J.E.: Astron. J. 114, 1992 (1997)

Reipurth, B., Bally, J., Aspin, C., Connelley, M.S., Geballe, T.R., Kraus, S., Appenzeller, I., Burgasser, A.: Astron. J. 146, 118 (2013)

Reipurth, B., Clarke, C.J., Boss, A.P., Goodwin, S.P., Rodriguez, L.F., Stassun, K.G., Tokovinin, A., Zinnecker, H.: ArXiv e-prints (2014). 1403.1907 
Reipurth, B., Guimarães, M.M., Connelley, M.S., Bally, J.: Astron. J. 134, 2272 (2007)

Rizzuto, A.C., Ireland, M.J., Robertson, J.G., Kok, Y., Tuthill, P.G., Warrington, B.A., Haubois, X., Tango, W.J., Norris, B., ten Brummelaar, T., Kraus, A.L., Jacob, A., Laliberte-Houdeville, C.: Mon. Not. R. Astron. Soc. 436(2), 1694 (2013)

Roddier, C., Roddier, F., Northcott, M.J., Graves, J.E., Jim, K.: Astrophys. J. 463, 326 (1996)

Rodriguez, D.R., Zuckerman, B.: Astrophys. J. 745, 147 (2012)

Rosenfeld, K.A., Andrews, S.M., Wilner, D.J., Stempels, H.C.: Astrophys. J. 759, 119 (2012)

Shevchenko, V.S., Vitrichenko, E.A.: In: The, P.S., Perez, M.R., van den Heuvel, E.P.J. (eds.) The Nature and Evolutionary Status of Herbig Ae/Be Stars. Astronomical Society of the Pacific Conference Series, vol. 62, p. 55 (1994)

Shevchenko, V.S., Grankin, K.N., Ibragimov, M.A., Kondratiev, V.B., Melnikov, S.Y.U., Petrov, P.P., Shcherbakov V.A., Vitrichenko, L.A.: In: The, P.S., Perez, M.R., van den Heuvel, E.P.J. (eds.) The Nature and Evolutionary Status of Herbig Ae/Be Stars. Astronomical Society of the Pacific Conference Series, vol. 62, p. 43 (1994)

Siess, L., Dufour, E., Forestini, M.: Astron. Astrophys. 358, $593(2000)$

Smith, K.W., Balega, Y.Y., Duschl, W.J., Hofmann, K.H., Lachaume, R., Preibisch, T., Schertl, D., Weigelt, G.: Astron. Astrophys. 431, 307 (2005)

Stelzer, B., Robrade, J., Schmitt, J.H.M.M., Bouvier, J.: Astron. Astrophys. 493, 1109 (2009)

Testi, L., Palla, F., Natta, A.: Astron. Astrophys. 342, 515 (1999)

The, P.S., de Winter, D., Perez, M.R.: Astron. Astrophys. Suppl. Ser. 104, 315 (1994)

Thomas, S.J., van der Bliek, N.S., Rodgers, B., Doppmann, G., Bouvier, J.: In: Hartkopf, W.I., Harmanec, P., Guinan, E.F. (eds.) IAU Symposium. IAU Symposium, vol. 240 , p. 250 (2007)

Trilling, D.E., Stansberry, J.A., Stapelfeldt, K.R., Rieke, G.H., Su, K.Y.L., Gray, R.O., Corbally, C.J., Bryden, G., Chen, C.H., Boden, A., Beichman, C.A.: Astrophys. J. 658, 1289 (2007)

Vieira, S.L.A., Corradi, W.J.B., Alencar, S.H.P., Mendes, L.T.S., Torres, C.A.O., Quast, G.R., Guimarães, M.M., da Silva, L.: Astron. J. 126(6), 2971 (2003)

Vink, J.S., Drew, J.E., Harries, T.J., Oudmaijer, R.D., Unruh, Y.: Mon. Not. R. Astron. Soc. 359, 1049 (2005)

Wheelwright, H.E., Oudmaijer, R.D., Goodwin, S.P.: Mon. Not. R. Astron. Soc. 401, 1199 (2010)

Wheelwright, H.E., Vink, J.S., Oudmaijer, R.D., Drew, J.E.: Astron. Astrophys. 532, 28 (2011)

Yasui, C., Kobayashi, N., Tokunaga, A.T., Saito, M.: Mon. Not. R. Astron. Soc. 442, 2543 (2014)

Zinnecker, H., Preibisch, T.: Astron. Astrophys. 292, 152 (1994)

This manuscript was prepared with the AAS LATEX macros v5.2. 